\title{
Rapid, Comprehensive Analysis of the Dystrophin Transcript by a Custom Micro-Fluidic Exome Array
}

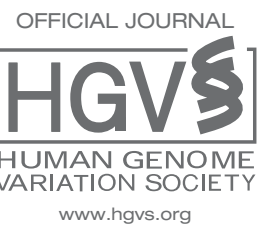

\author{
Matteo Bovolenta, ${ }^{*}$ Chiara Scotton, Maria Sofia Falzarano, Francesca Gualandi, and Alessandra Ferlini* \\ Department of Experimental and Diagnostic Medicine, Section of Medical Genetics, University of Ferrara, Ferrara, Italy \\ Communicated by Christophe Béroud \\ Received 14 July 2011; accepted revised manuscript 20 December 2011. \\ Published online 29 December 2011 in Wiley Online Library (www.wiley.com/humanmutation).DOI: 10.1002/humu.22017
}

\begin{abstract}
Duchenne and Becker muscular dystrophies are caused by mutations in the dystrophin gene. Both the enormous size of this gene and heterogeneous set of causative mutations behind these pathologies may hamper and even prevent accurate molecular diagnosis. Often RNA analysis is required not only to identify mutations escaping MLPA/CGH or exon sequencing but also to validate the functional effect of novel variations that may affect the exon composition of the DMD gene. We present the design and experimental validation of a new, simple, and easy-to-use platform we call FluiDMD. This platform is based on the Applied Biosystems 7900HT TaqMan ${ }^{\circledR}$ low-density array technology and is able to define the fullexon composition, profile the dystrophin isoforms present, establish changes in mRNA decay, and potentially identify all deletions/duplications and splicing affecting mutations contemporaneously. Moreover, we demonstrate that this system accurately detects the pathogenic effect of all dystrophin mutations belonging to any category, thereby highlighting the functional validation capacity of this system. The high efficacy and sensitivity of this tool in detecting mutations in the dystrophin transcript can be exploited in a variety of cells/tissues, in particular skin, which is harvested by causing minimum patient discomfort. We therefore propose FluiDMD as a validated diagnostic biomarker for molecular profiling of dystrophinopathies.

Hum Mutat 33:572-581, 2012. (C) 2011 Wiley Periodicals, Inc.
\end{abstract}

KEY WORDS: diagnostic biomarker; DMD mutations; fluidic cards; RNA analysis

\section{Introduction}

Duchenne (DMD; MIM\# 310200) and Becker (BMD; MIM\# 300376) muscular dystrophies are muscle-wasting diseases caused, respectively, by out-of-frame and in-frame mutations in the dys-

\footnotetext{
Additional Supporting Information may be found in the online version of this article. *Correspondence to: Matteo Bovolenta, Department of Experimental and Diagnostic Medicine, Section of Medical Genetics, University of Ferrara, 74 Fossato di Mortara, Ferrara 44100, Italy. E-mail: bvlmtt@unife.it or Alessandra Ferlini, Department of Experimental and Diagnostic Medicine, Section of Medical Genetics, University of Ferrara, 74 Fossato di Mortara, Ferrara 44100, Italy E-mail: fla@unife.it

Contract grant sponsor: BIO-NMD (EC, 7th FP, proposal \#241665; www.bio-nmd.eu, to $\mathrm{AF}$ as coordinator).
}

trophin gene [Muntoni et al., 2003]. In 1988, Monaco observed that DMD patients typically show loss of dystrophin protein expression due to frame-shifting mutations, while BMD patients maintain the reading frame and produce a reduced quantity of semifunctional dystrophin, a mechanism known as the "reading-frame rule" [Monaco et al., 1988].

However, an increase in the precision of dystrophinopathy diagnosis in recent years has led to the conclusion that this rule applies mainly to DMD and out-of-frame mutations, but only to about half of patients with BMD [Flanigan et al., 2009; Kesari et al., 2008]. Both in-frame and out-of-frame mutations in the DMD gene (MIM\# 300377) belong to a very heterogeneous mutation spectrum; in $75-80 \%$ of all cases this involves deletions/duplications (pathogenic copy number variations or CNVs), whereas the remaining 20-25\% feature small mutations. About $2 \%$ of dystrophin mutations are atypical, and are generally due to deep intronic mutations that affect the splicing choices of the gene [Abbs et al., 2010].

Deletions and duplications are easily detected by diagnostic procedures such as multiple ligase probe amplification (MLPA) [Janssen et al., 2005] or comparative genomic hybridization (CGH) [del Gaudio et al., 2008], the latter may be the most accurate since it also identifies pure intronic rearrangements. However, due to the considerable size of the DMD gene (79 exons), identification of small mutations is a highly challenging task, as extensive analysis of all of the exons, splicing borders, and regulatory sites needs to be performed by direct sequencing, single condition amplification/internal primer sequencing [Flanigan et al., 2003] or denaturing high-performance liquid chromatography [Bennett et al., 2001]. In addition, a few mutations escape detection by all of these techniques, thereby necessitating the combined use of CGH and RNA analysis, which requires the availability of patients' muscle biopsy. Unfortunately, the issue is further complicated by the fact that duplications or small mutations may have unpredictable effects on the behavior of RNA, influencing splicing events/choices and thereby impacting on the phenotype and genetic prognosis of the disease [Flanigan et al., 2011; Gualandi et al., 2009; Magri et al., 2011]. As described in Flanigan et al., 2011, the prediction of the effect of a mutation could be improved by using bioinformatic tools, but still a few cases remain undisclosed. Hence, definition of the RNA profile is recommended for establishing the genetic prognosis in DMD gene mutations (especially duplications or small mutations of uncertain significance), a fact which is of particular relevance to the forthcoming and ongoing therapeutic trials exploring novel, often mutation-specific approaches.

Thus, the pathogenic effect of many mutations often necessitates their validation by RNA analysis using RT-PCR and sequencing [Deburgrave et al., 2007], a time-consuming and laborious task. However, an attempt to overcome these difficulties via the application of MLPA to cDNA was made by Kesari et al. [Kesari 
et al., 2008], in a work that evidenced the notable exception to the reading-frame rule in $\mathrm{BMD}$ patients in $\mathrm{gDNA}, \mathrm{cDNA}$, and protein studies. In the same study, the MLPA/cDNA approach was shown to be useful in detecting deletions and splicing mutations, but was unfortunately not so successful in detecting duplications at the cDNA level.

More recently, the advent of targeted sequencing by Next Generation Sequencing platforms ( 454 technology) facilitated the detection of small mutations in the DMD gene. Nevertheless, this approach is expensive, time consuming, and fails to identify CNVs [Bonnal et al., 2010], and, furthermore, will require a certain amount of further research and validation in order to be fit for purpose.

Here, however, we propose a new, accurate, and rapid gene/ exome-specific method based on Applied Biosystems 7900HT Micro Fluidic Card technology. This novel platform, the FluiDMD, is able to simultaneously analyze 85 unique TaqMan Real-Time systems, designed to recognize 76 of 78 DMD exon junctions, all DMD isoforms, and two endogenous controls, consenting detect of both $\mathrm{del} /$ dup and small mutations affecting the exon composition, a considerable step forward with respect to gMLPA, cDNA-MLPA, and CGH.

Moreover, we were able to validate this new diagnostic method by detecting mutations both previously identified by standard procedures and not yet diagnosed. In one case, we were also able to explain the pathogenic effect of a deep intronic duplication identified by CGH. RT-PCR RNA analysis confirmed the FluiDMD card results, thereby validating the potential of this innovative diagnostic tool. Interestingly, FluiDMD were able to identify mRNA decay changes of the DMD transcript in patients with nonsense or out-of-frame mutations. In addition, the FluiDMD technique was successfully applied to RNA extracted from a variety of patients' tissues (muscle, skin, and cells), thereby possibly obviating the need for the highly invasive procedures such as muscle biopsy often required by current approaches.

To our knowledge, this is the first report of a gene-specific TaqMan ${ }^{\circledR}$ Low Density Array (TLDA) able to completely characterize the transcript of a gene as large as DMD.

\section{Materials and Methods}

\section{Patients}

Twenty three patients (males) with DMD/BMD and one control (male) were subjected to testing by the FluiDMD TLDA, after informed consent was obtained during the course of routine diagnostic procedures. All patients provided muscle tissue and, in four cases, myogenic cells (either myoblasts or MyoD-transformed fibroblasts) and 14 were previously characterized by MLPA, CGH, or DNA sequencing, as reported in Table 1 . To validate the range of efficacy of the FluiDMD card, four patients with duplications (exons 2, 14-22, 18-42, 34-41), three with deletions (exons 52, 2-44, 45-50) and three with intronic mutations affecting the exon composition (c.1705-5T $>\mathrm{G}$ in intron $14, \mathrm{c} .10223+2 \mathrm{~T}>\mathrm{C}$ in intron 70 , and a deep intronic duplication of $1.4 \mathrm{~kb}$ in intron 4 ) were selected.

To demonstrate the ability of the FluiDMD to identify the decay of the DMD transcript in patients with nonsense or out-of-frame mutations, it was applied to two patients with stop-codon mutations (p.E1150X in exon 26 and p.Q983X in exon 22) and two patients with single-exon duplications in exon 12 (out-of-frame) and 16 (in-frame), respectively.

The FluiDMD's ability to detect mutation in blind diagnostic conditions was assessed by testing nine patients with a clinical diag- nosis of DMD/BMD but uncharacterized at molecular level (Table 1: T1-T9).

Finally, to confirm our hypothesis that this assay can also detect the dystrophin transcript in tissues harvested by low-invasive procedures, we successfully validated the FluiDMD on RNA obtained from skin biopsy from two controls and one DMD patient.

Nucleotide positions were determined according to the reference DMD sequence used for mutation analysis (GenBank accession number NM_004006.2); nucleotide numbering reflects cDNA numbering with +1 corresponding to the A of the ATG translation initiation codon in the reference sequence, according to journal guidelines (www.hgvs.org/mutnomen). The initiation codon is codon 1 .

\section{RNA Extraction}

Total RNA was isolated from muscles, skin biopsies, and cells using the RNeasy Kit (Qiagen, Valencia, CA), and reverse-transcribed by means of a High-Capacity cDNA Reverse Transcription Kit (Applied Biosystems, Foster City, CA), according to the manufacturer's instructions (200 ng RNA for muscle and cells, 400 ng RNA for skin).

In Patients 4 and 6, total RNA was isolated from cultured myoblasts, whereas in Patient 7 and T1, total RNA was obtained from Myo-D-transformed fibroblasts, as previously described [Spitali et al., 2009]. Before cDNA synthesis, RNA was treated with DNAse I (Roche, Branford, CT) and checked for residual DNA contamination by a 55-cycle PCR.

\section{DMD Gene Micro Fluidic Card (FluiDMD) Protocol and Analysis}

A technical and conceptual description of the TaqMan ${ }^{\circledR}$ LowDensity Array based on an Applied Biosystems (ABI) 7900HT Micro-Fluidic Card together with the relevant protocol can be found in the Applied Biosystems TaqMan ${ }^{\circledR}$ Array Micro-Fluidic Cards User Guide (http://www3.appliedbiosystems.com/cms/groups/mcb _support/documents/generaldocuments/cms_062836.pdf). However, for explanatory purposes a brief description of the protocol is provided here: $200 \mathrm{ng}$ of RNA from each sample is retrotranscribed using High-Capacity cDNA Reverse Transcription Kit (Applied Biosystems) in a volume of $20 \mu \mathrm{l}$. To this quantity is added 100 $\mu \mathrm{l}$ of $2 \times$ Universal Master mix (Applied Biosystems) and $80 \mu \mathrm{l}$ of sterile water. $100 \mu \mathrm{l}$ per port of the resulting mixture is loaded onto the fluidic cards. Before these are sealed, two 1-min runs on a Sorvall centrifuge are performed at $1200 \mathrm{rpm}$ (ramp rates set at 9). Finally, the Micro Fluidic Cards are run on a Real-Time 7900HT appliance (Applied Biosystems). The raw data obtained from FluiDMD analysis is available in the Supp. Table S1.

\section{Mutation Analysis}

In the case of duplications, data analysis was performed by adapting the $\Delta \Delta \mathrm{CT}$ Method to our system (Applied Biosystems User Bulletin \#2). A variation in the representation of an exon junction was evaluated by subtracting the CT of each system from all the others in both the test and control samples. The total $\Delta \mathrm{CT}$ s in the test sample thereby obtained were subtracted from the corresponding $\Delta \mathrm{CTs}$ in the control sample. Thus, the amount of target sequence, normalized to all of the systems present in the fluidic assay, is given by the mean of all the $\Delta \Delta$ CTs of a single system elevated by $2^{-\Delta \Delta C T}$, and is represented in a dot-to-dot graph (Fig. 1). 


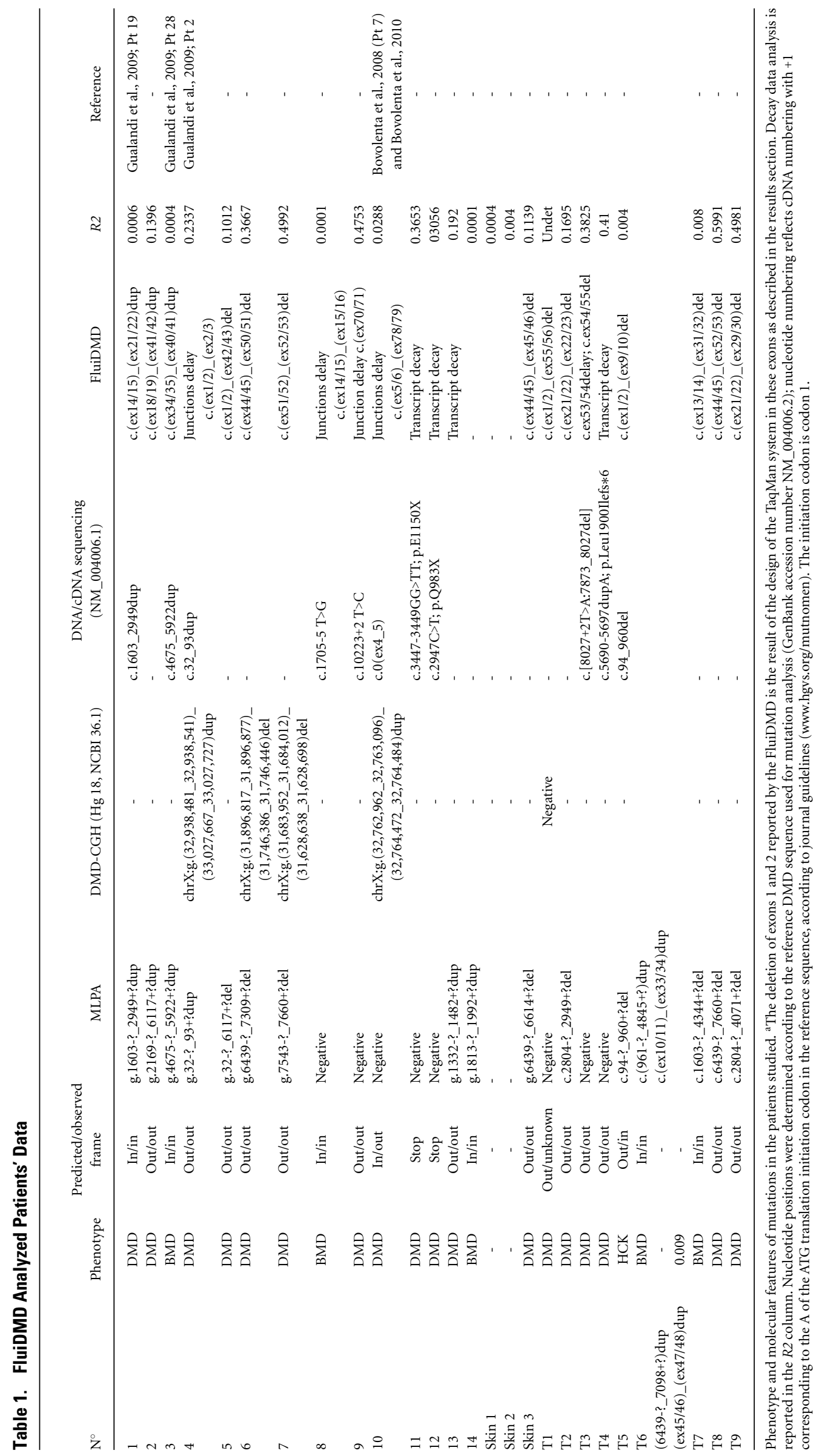



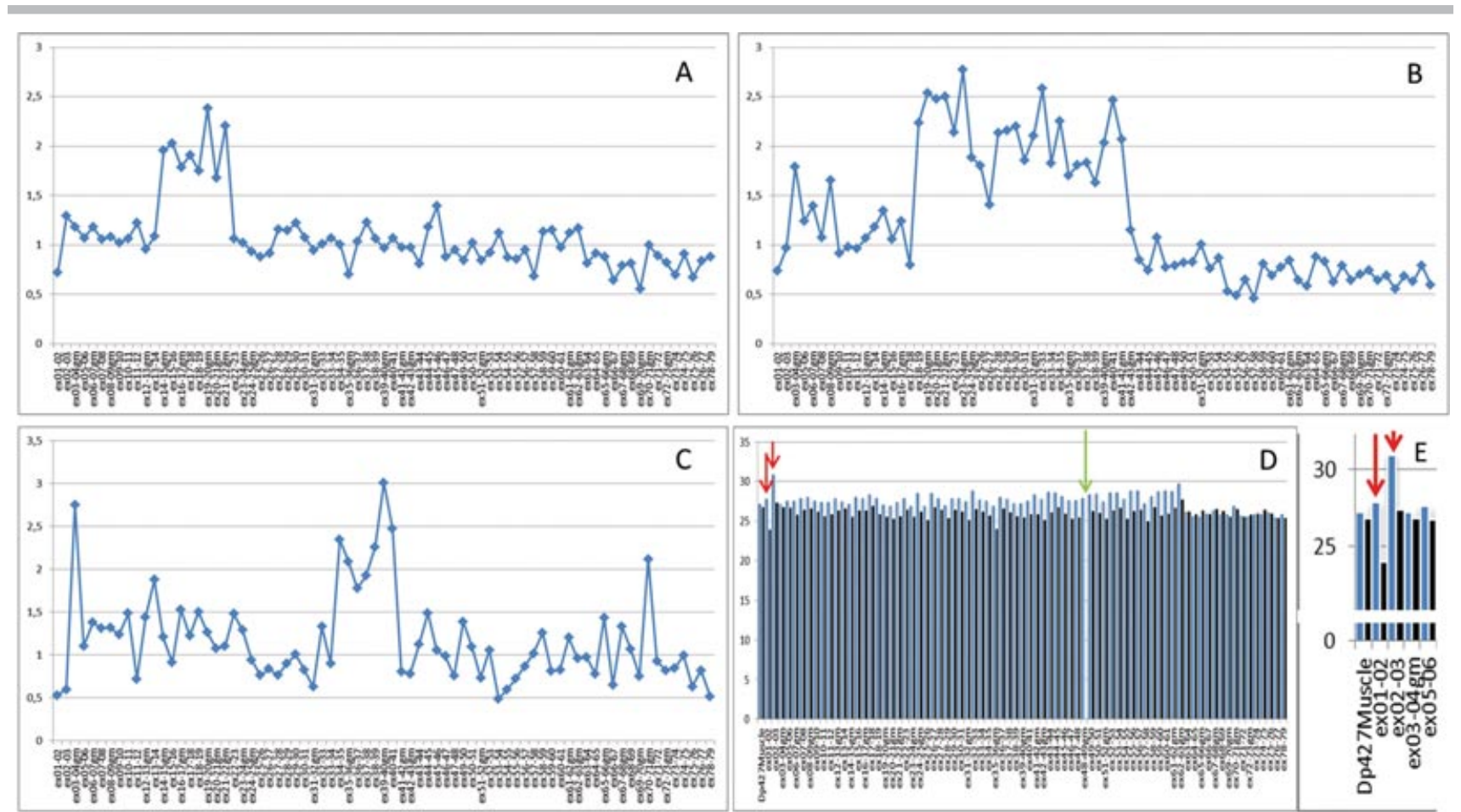

Figure 1. FluiDMD profiles of four duplications. Duplication detection was performed by subtracting the CT of each system from all of the others in both control and test samples. All $\Delta$ CTs in the test sample thereby obtained were subtracted from the corresponding $\Delta$ CTs in the control sample. Thus, the amount of target sequence, normalized to all the systems present in the fluidic array, is given by the mean of all the $\Delta \Delta$ CTs of a single system elevated by $2^{-\Delta \Delta C T}$, and is represented on a dot-to-dot graph showing duplications from exon 14-22 (A, Patient 1), from exon 18-42 (B, Patient 2), and between exons 34 and 41 (C, Patient 3). Red/dark gray arrows in D and E (zoom in) show the delayed ex1-ex2 and ex2-ex3 systems in Patient 4 (carrying an exon-2 duplication) with respect to the profile of the control sample (black bars). The presence of the SNP rs1800274 in the control resulted in the failure to amplify the junction ex48-49 (green/ light gray arrow). The CT values from exon 63-79 represent the portion of fibroblasts expressing Dp 71 isoform in the myoblast culture from Patient 4. [Color figure can be viewed in the online issue, which is available at wileyonlinelibrary.com.]

Deletions were easily displayed by representing the CTs for each junction as a histogram (Figs. 2, 3, and 4).

\section{Decay Analysis}

To estimate the decay of the DMD transcript, a histogram was created with a spreadsheet using the raw CT values for each sample tested, without taking into account the regions affected by the mutations. A linear regression line was drawn and the $R^{2}$ calculated (Table 1 and Supp. Fig. S1).

\section{DMD Gene Isoform Expression Analysis}

Relative quantification of the DMD gene isoforms was performed by creating two patient groups: the first (Patients $1-3,5,8-10$ ) donated muscle tissue for analysis and the second cultured myoblasts or MyoD-transformed fibroblasts (Patients 4, 6, and 7). In each group, the mean CT value relative to the systems specifically designed for the DMD gene isoforms was subtracted from the mean CT value of the Dp $427 \mathrm{~m}$ isoform systems. The results were elevated by $2^{-\Delta \Delta \mathrm{CT}}$ and are shown as a histogram (Fig. 5).

\section{Validation by RT-PCR and Sequencing}

Validation of the results obtained by the FluiDMD card was obtained by RT-PCR and sequencing as previously described [Gualandi et al., 2009].
Sequencing has previously been reported in Patients 1, 4, and 10 [Bovolenta et al., 2008; Bovolenta et al., 2010; Gualandi et al., 2009].

In Patients 2 and 3, duplication breakpoints were amplified and sequenced using a forward primer on the second-to-last duplicated exon and a reverse primer on the second-duplicated exon (Ex41FEx19R for dup 18-42 and Ex40F-Ex33R for dup 34-41).

In Patients 5, 6, and 7, deletion breakpoints were amplified and sequenced using a pair of oligonucleotides located outside the first and last deleted exons (Ex1F-ex45R, Ex44F-Ex51R, and Ex51FEx53R, respectively, for exons 2-44, 45-50, and 52 deletions).

In Patients 8 and 9, exons 11-16 and 67-71 were respectively amplified and sequenced using primers Ex11F-Ex16R and Ex67FEx71R.

Validation of the mutations identified in patients T3 and T5 was performed by amplifying the regions corresponding to exons from 51 to 57 and from 1 to 11 , respectively. For patient T4, the amplification of all the Dp427m isoform was required to identify the disease-causing mutation.

\section{Validation by MLPA}

MLPA analysis (SALSA MLPA KIT P034/P035 from MRCHolland, Amsterdam, the Netherlands) was performed as previously described [Lalic et al., 2005] to validate the results obtained from the nine undiagnosed patients where deletions or duplications were identified by FluiDMD assay. 

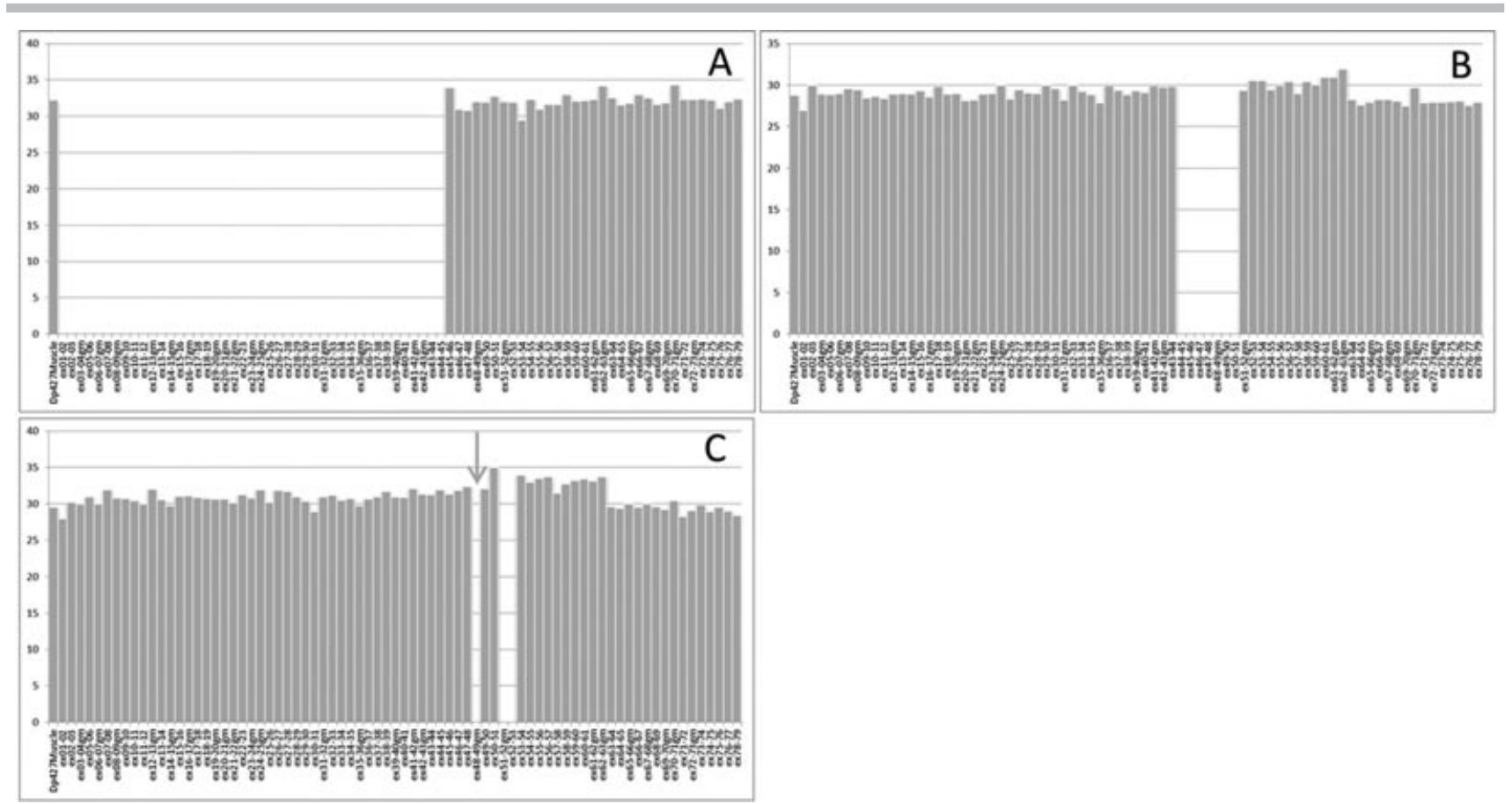

Figure 2. FluiDMD profiles of three deletions. CT values for each system were ranked from exon 1-79, and displayed on a histogram together with the Dp427m system. Deletions identified were: from exon 2-44 (A, Patient 5), from exon 45-50 (B, Patient 6), and exon 52 (C, Patient 7). In B and C, RNA for the analysis was extracted, respectively, from cultured myoblasts and MyoD-transformed fibroblasts. In both graphs, the CT values from exon junction 63-64 highlight the presence of fibroblasts expressing the Dp71 isoform. The presence of the SNP rs1800274 in Patient 7 resulted in the failure to amplify the junction ex48-49 (arrow).
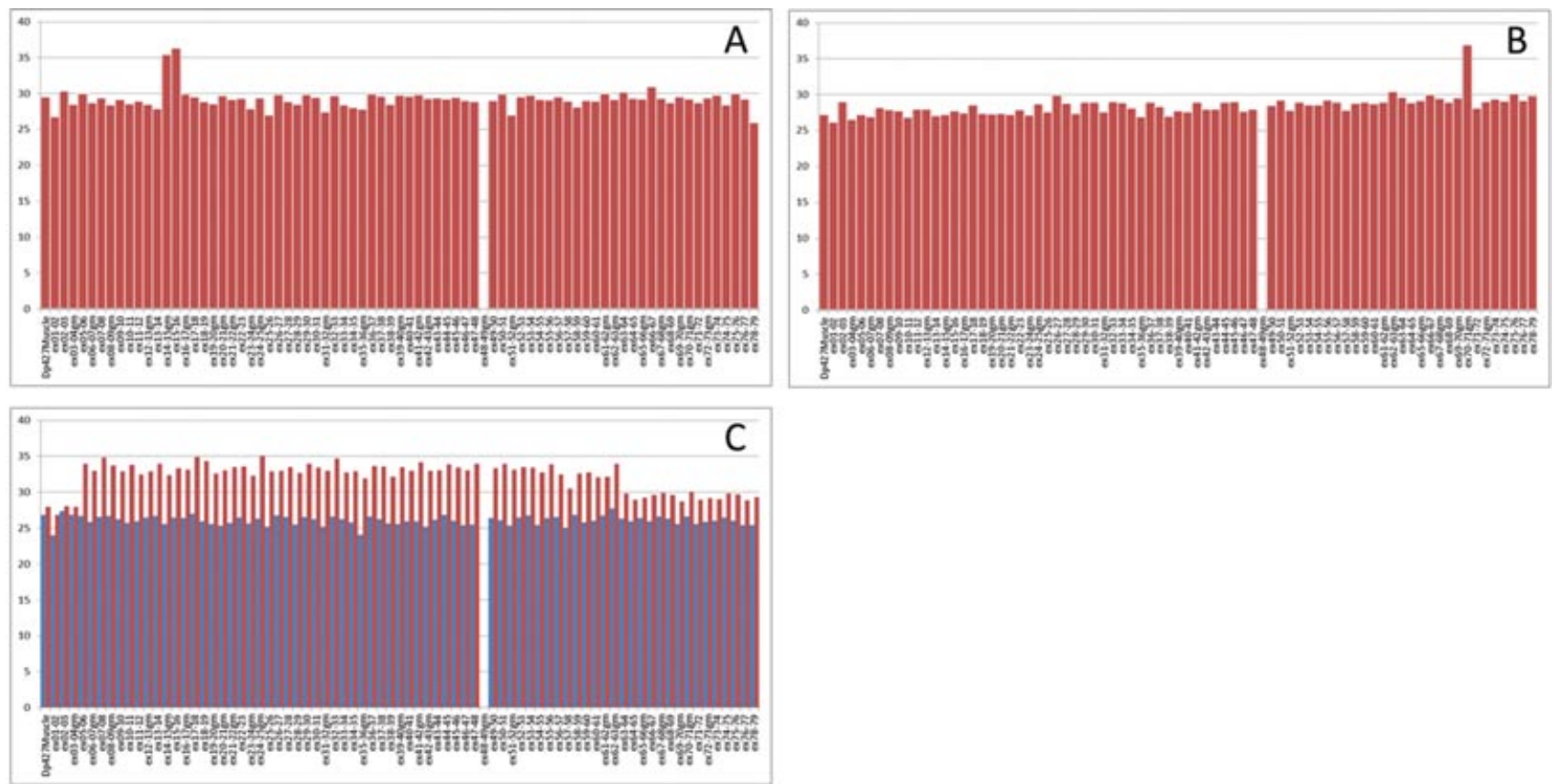

Figure 3. FluiDMD profiles of three mutations affecting exon composition. In Patient $8(A)$, carrying a $T>G$ transversion at position -5 of $D M D$ intron 14, we found a delay of about seven CTs with respect to the mean of all the other systems at exon junctions 14-15 and 15-16, suggesting poor incorporation of exon 15. Patient 9 (B) had a $T>C$ transition in the canonical donor splice site of intron 70, and showed a delay in the single-exon junction between exon 70-71. The last Patient $(10, C)$ carries a duplication within intron 4 that resulted in the retention of the intron itself and consequently the identification of an in-frame stop codon located at nucleotide c.264+34. The FluiDMD revealed a delay of 6 units in the CT values from exon junctions 5-6 to 62-63 and of 3 units from exon junctions $63-64$ to 78-79. [Color figure can be viewed in the online issue, which is available at wileyonlinelibrary.com.] 

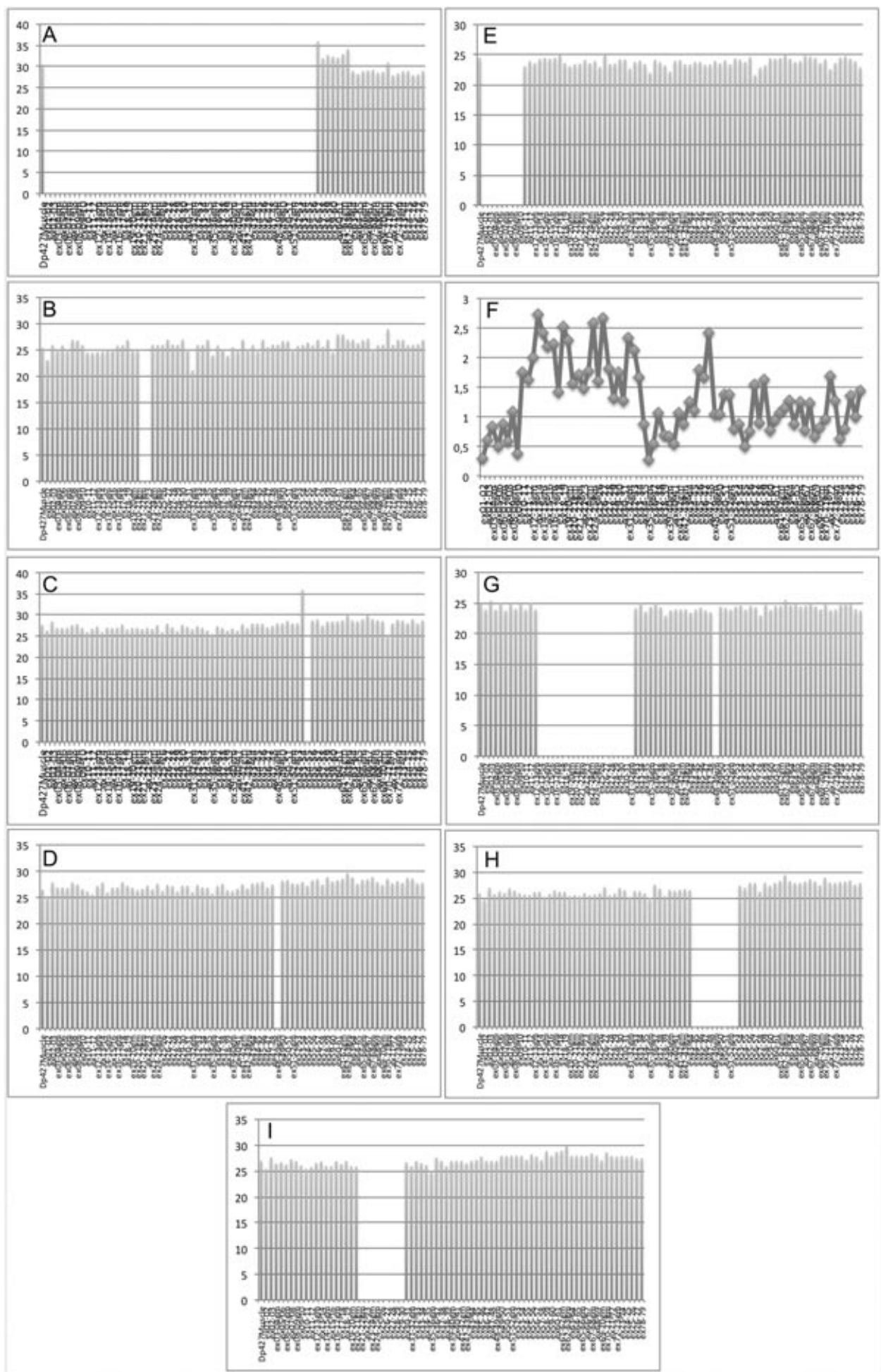

Figure 4. FluiDMD profiles of nine undiagnosed patients. In patients $T 1(A), T 2(B)$, $T 5(E), T 7(G)$, $T 8(H)$, and T9 (I), the FluiDMD identified the absence of amplification of exon junctions from $1 / 2$ to $55 / 56$, from $21 / 22$ to $22 / 23$, from $1 / 2$ to $9 / 10$, from $13 / 14$ to $31 / 32$, from $44 / 45$ to $52 / 53$, and from $21 / 22$ to $29 / 30$, respectively. The junctions between the exons $53 / 54$ and $54 / 55$ were respectively delayed and absent in patient T3 (C). The FluiDMD mutation analysis in patient T4 (D) found no mutations. Patient T6 (F) was found to carry two non-contiguous duplication from exon junctions 10/11 to $33 / 34$ and from $45 / 46$ to $47 / 48$. 


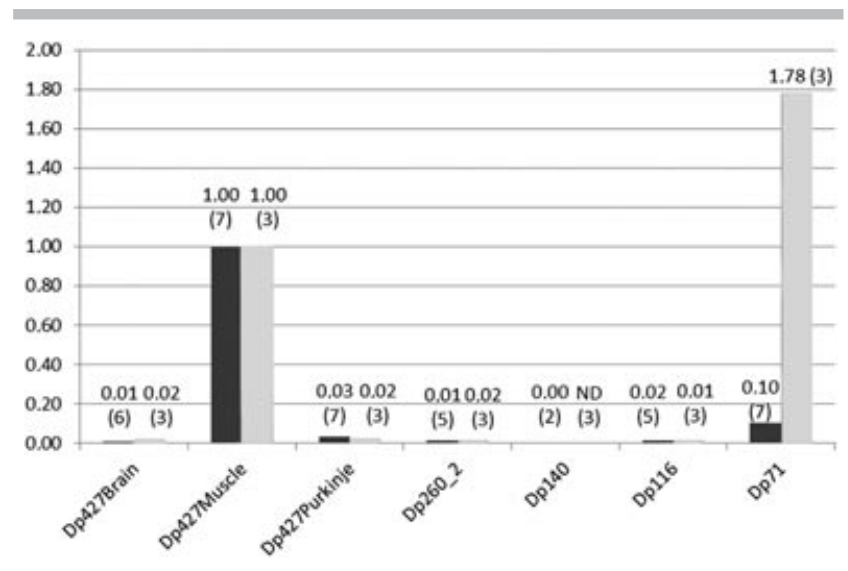

Figure 5. DMD isoforms analysis. Relative quantification of the DMD gene isoforms performed in patients' muscles (dark gray histograms) and cultured cells (light gray histograms; myoblasts, and Myo-Dtransformed fibroblasts). The number of patients in which each isoform was detected is shown in parentheses above each histogram. Dp427m isoform was set as one, and the other isoforms were quantified in relation to it. Dp427b, Dp427p, Dp260, and Dp116 were barely detectable as a result of illegitimate usage of these promoters. Dp140 was almost absent in all samples.

\section{RESULTS}

\section{FluiDMD Design}

By combining the 51 already available ABI real-time exon-exon junction systems with 25 systems specifically designed by Primer Express v2.0 to cover the exon-exon junctions not provided by Applied Biosystems, we created the gene-specific fluidic card FluiDMD (Supp. Table S2).

Whenever possible, exon junction systems meeting the following standards were selected: absence of possible homology with other sequences; probes, forward or reverse primers designed so that the junction between two exons overlapped part of its sequence; absence of SNPs (Single Nucleotide Polymorphism) in the primers and probe sequences.

These criteria made it impossible to design exon junction systems between junctions $4 / 5$ and $77 / 78$, and the forward primer in the 48/49-junction system was useless due to the presence of a known SNP. Moreover, due to the limited size of exons 1 and 2, the Applied Biosystems assay for this junction is not a pure exon-exon junction as the reverse primer is located in exon 3.

In order to reach the custom assay format, 96a, which comprises of 94 unique assays plus one mandatory control replicated twice and eight unique port sets, we added human ACTB and 18S human assays as housekeeping assays. To fill the fluidic card, we designed specific Taqman ${ }^{\circledR}$ systems for the DMD gene isoforms and replicated our previously designed real-time systems.

Each port distributed the sample to 48 different 2 - $\mu$ l wells containing lyophilized Taqman ${ }^{\circledR}$ systems. In order to analyze the full DMD transcript, one sample was loaded twice in two consecutive ports, analyzing roughly 2 ng of total RNA retrotranscribed to cDNA per well.

\section{FluiDMD Mutation Analysis}

We first validated our FluiDMD card by analyzing four patients (Patients 1, 2, 3, and 4) with various degrees of duplication previ- ously identified by MLPA, these mutations being the most difficult to identify using standard diagnostic procedures (Fig. 1). RNA analysis was performed on Patient 1, who had previously been characterized by MLPA, in an attempt to explain the discrepancy between the DMD phenotype and the in-frame duplication [Gualandi et al., 2009].

In this study, we determined both the scope of the duplication, involving exon junctions from $14-15$ to $21-22$, and the absence of skipping events within it (Fig. 1A), thereby supporting the hypothetical implication of post-transcriptional mechanisms in the pathogenesis. Furthermore, in Patients 2 and 3, the FluiDMD card precisely identified the respective exon duplications 18-42 and 3441 (Figs. 1B and C). However, the quality of the RNA (due to the different extraction dates) influenced the profile of some systems in these two samples (e.g., 3-4, 8-9, 26-27 in Patient 2 and 3-4, 13-14, $70-71$ in Patient 3).

Patient 4's exon-2 duplication was returned by the FluiDMD as a delay in the CT value of exon junctions $1-2$ and 2-3 (Figs. 1D and E). This was presumably due to competition between the two species generated by the annealing of one of the two primers in the system to the duplicated regions.

Three patients $(5,6$, and 7$)$ featuring deletions involving different exons were used to test the efficacy of the FluiDMD in identifying these kinds of rearrangements. All patients' deletions were identified by MLPA, which revealed the loss of exons from 2 to 44,45 to 50, and 52 in Patients 5, 6, and 7, respectively. The TLDA system we designed failed to amplify the exon junction systems corresponding to the deletions in all cases (Figs. 2A-C).

A final group of patients was selected, because RNA analysis was required to validate the pathogenicity in these cases, to determine the ability of the FluiDMD card to detect mutations affecting the normal exon composition. Thus, two patients with point mutations in the splice site consensus sequences (Patients 8 and 9) and a deep intronic duplication shown to induce intronic retention (Patient 10, see Bovolenta et al., 2008) were chosen.

In the first case, Patient 8 was found to carry a $\mathrm{T}>\mathrm{G}$ transversion at position -5 of DMD intron 14 (nucleotide c.1705-5 of the DMD gene cDNA reference sequence NM_004006.1). FluiDMD analysis in this patient revealed a delay of roughly seven CTs in the exon junctions 14-15 and 15-16 with respect to the mean of all the other systems (Fig. 3A), thereby suggesting an impediment to efficient incorporation of exon 15.

Patient 9 was selected for a $\mathrm{T}>\mathrm{C}$ transition in the canonical donor splice site of intron 70 (position c.10223+2), which should impair the recognition of the $3^{\prime}$ on the same exon. Analyzed by means of the FluiDMD card, Patient 9 showed a delay in the single-exon junction between exon 70-71, thereby confirming the hypothesis. (Fig. 3B).

Patient 10 was previously described as possessing a duplication within intron 4 , resulting in the retention of the intron itself and the consequent identification of an in-frame stop codon located at nucleotide c.264+34 [Bovolenta et al., 2008; Bovolenta et al., 2010]. The FluiDMD revealed delays of 6 units in the CT values from exon junctions 5-6 to 62-63, and of 3 units from exon junctions 63-64 to $78-79$ (Fig. 3C), thereby confirming intron retention and highlighting a very high decay with a strong transcriptional impairment of regions distal to the mutation.

Further analysis of nine undiagnosed DMD/BMD patients revealed the presence of deletions in six cases (T1, T2, T5, T7, T8, and T9), duplications in patient T6, delay and absence of two consecutive systems in patient T3. No mutations were identified in patient T4 (Fig. 4 and Table 1).

The presence of the rs1800274 SNP $(A>G)$ in the fourth-to-last nucleotide of the forward primer for the system $48-49$ resulted in 
failure to amplify this junction in Patients 7-10, T4, T7, and the control. In addition, the expression of the isoform Dp71 (from exon 63-79), due to the presence of fibroblasts, was identified in the cultures of Patients 4, 6, 7, and T1 (Figs. 1D, 2B, and C4).

\section{Decay Analysis}

Cursory examination of the histograms revealed that the CT values in some patients increased from the $5^{\prime}$ to the $3^{\prime}$ of the gene in relation to the frame of the DMD transcript. In fact, by drawing a linear regression line and calculating the $R^{2}$, we registered values very close to zero for in-frame mutations and higher than 0.1 for out-of-frame mutations (Table 1). Similar behavior was observed in patients with stop mutations and out-of-frame single-exon duplications (Table 1). The only exception to this rule is the intermediate $0.028 R^{2}$ value of Patient 10 that is influenced by the behavior of the TaqMan systems downstream the mutation in this patient (see Table 1 and Fig. 3C).

Also in the nine undiagnosed patients, the decay analysis showed correlation with the mutations identified resulting in low $R^{2}$ values in the two BMD (T6 and T7) and one HCK (High Creatin Kinase) (T5) patients and high $R^{2}$ values in the other patients. This implies that either a stop mutation or single-exon duplication should be present in patient $\mathrm{T} 4$ as no mutations were identified by the FluiDMD.

\section{FluiDMD Isoform Analysis}

Relative quantification of the DMD isoforms in muscle tissue and cultured cells (myoblasts and Myo-D-transfected fibroblasts) revealed contrasting results. Indeed, muscle analysis showed the presence of Dp427m, Dp427p, and Dp71 isoforms in all (seven) samples, Dp427b in six patients, Dp240 and Dp116 in five patients borderline levels of Dp140 in two (Fig. 4), whereas in cultured myogenic cells, the expression of all isoforms was detected in all patients with the exception of Dp140.

The differing composition in terms of cell types was evidenced by the different quantities of Dp71 detected in the two groups: from 0.10 to 1.78 times with respect to $\mathrm{Dp} 427 \mathrm{~m}$ in muscle and the cultured cells, respectively. This result is consistent with the sub-population of fibroblasts that are known to be present in these cultures (Fig. 5).

In both groups, only Dp427m and Dp71 showed strong signals, while the other isoforms were expressed at very low levels, presumably due to the ectopic/illegitimate activation of these promoters.

\section{Validation by RT-PCR and Sequencing}

The duplication breakpoints of Patients 2 and 3 were amplified and sequenced, resulting in the detection of a junction between exons 42 and 18 in the first case, and a junction between exons 41 and 34 in the latter, thereby confirming the accuracy of the results obtained with FluiDMD and MLPA (data not shown).

Amplification and sequencing of the deletion breakpoints in $\mathrm{Pa}$ tients 5, 6, and 7 detected the junctions between exons 1-45, 44-51, and 51-53, respectively (data not shown).

RT-PCR in Patient 8 confirmed the skipping of exon 15 due to the mutation identified at the DNA level (Supp. Fig. S2A).

RT-PCR in Patient 9 amplified two different products; sequencing of the higher product revealed the presence of a transcript with a correct junction between exons 69 and 70, but recognized a cryptic donor splice site within exon 70 (leading to the loss of $101 \mathrm{nu}$ cleotides at the $3^{\prime}$ of exon 70) before its junction with exon 71 (Supp. Fig. S2B). The sequence of the second product, on the other hand, showed the complete absence of exon 70 (Supp. Fig. S2C). Both products resulted in the recognition of a premature stop codon at position c.3432, within exon 72 .

RT-PCR in patient T3 showed the amplification of a single product of reduced weight due to the absence of exon 54. Genomic DNA amplification and sequencing of exon 54 identified a $\mathrm{T}>\mathrm{A}$ transversion in the donor splicing site (data not shown). cDNA amplification and sequencing of patient T5 identified the junction between exons 2 and 10 as a result of an in-frame deletion of exons 3-9 (data not shown).

The full amplification of the Dp427m isoform in patient $\mathrm{T} 4$ found a duplication of a single adenine in exon 40 resulting in a frameshift and the recognition of a stop codon at position p. 1900 .

\section{Validation by MLPA}

MLPA analysis in patients T2 and T6-T9 validated the presence of both deletions and duplications identified with the FluiDMD system (data not shown). An exception was patient T5 where a deletion from exon 2-9 was detected with the TLDA whereas MLPA identified an exon 3-9 deletion (Table 1) confirming the RT-PCR results and suggesting the failure of the exon $1 / 2$ junction in the FluiDMD system due to the location of the reverse primer within exon 3.

Another exception was patient T1 where a deletion from exon 2-55 was detected with the FluiDMD card. MLPA analysis in this patient revealed no presence of deletions and duplications. Amplification of the breakpoint by RT-PCR and an attempt to detect possible breakpoints of an inversion by CGH gave negative results (data not shown), suggesting the possibility that a balanced rearrangement may be occurred in this patient.

\section{Skin Biopsy Analysis}

We have previously described the presence of dystrophin expression in the arrector pili smooth muscle (Ferlini et al., 2010), showing the potential of this muscle as a non-invasive biomarker in evaluating the efficacy of AON treatment. In order to analyze the dystrophin transcript from two controls and DMD skin biopsy samples, double the amount of RNA used for muscle analysis was loaded onto the FluiDMD card. In these tissues, we were able to correctly identify the transcription of the full-length Dp427muscle and the $3^{\prime}$ Dp71 isoforms and the DMD causing mutation (Supp. Table S1). In addition to the causing mutation, we found the absence of amplification of the junction between exon 19 and 20 due to a G-A transition within the probe sequence at the sixth nucleotide of exon 20 (data not shown).

\section{Discussion}

Diagnostic procedures for DMD have made a great leap forward with the development of MLPA and capillary sequencing, in terms of mutation detection rate for both del/dup and small mutations [Abbs et al., 2010; Flanigan et al., 2003; Janssen et al., 2005; Lalic et al., 2005]. More recently, the development of omic approaches, such as CGH and targeted resequencing, have heralded the arrival of rapid extensive screening for all types of mutation in this enormous gene [Bonnal et al., 2010; del Gaudio et al., 2008; Hegde et al., 2008].

Nonetheless, despite the great improvement in mutation detection at the genomic level, which will no doubt continue in the future with the validation of NGS (Next Generation Sequencing) techniques, diagnosis of dystrophinopathies often requires additional validation at the RNA level to establish the pathogenic impact of the 
genetic variations identified on the RNA phenotype. In fact, RNA analysis is considered an integral part of the diagnostic procedure for dystrophinopathies, as recently proposed in the best practice guidelines [Abbs et al., 2010]. Furthermore, since experimental DMD treatments, which are thought to act by dystrophin reframing, splice correction, or stop-codon reversion [Goemans et al., 2011; Kinali et al., 2009; Pichavant et al., 2011], are currently in progress, RNA profiling is still an unavoidable necessity at this stage.

Conventionally, RNA is sourced from muscle/myogenic cells obtained from patients through the highly invasive procedure of muscle biopsy. MyoD-transformed skin fibroblasts are an alternative, although myogenic cells do not represent a desirable source of RNA for diagnostic purposes, given the laborious nature of the culturing process, timing issues, and the limited amount of RNA that can be obtained [Chaouch et al., 2009].

However, herein we report both the design and validation of a $\mathrm{Hu}-$ man DMD TaqMan ${ }^{\circledR}$ Low-Density Array (or Micro-Fluidic Card) based on TaqMan ${ }^{\circledR}$ chemistry and the 7900 HT Sequence Detection System. Compared with standard reverse-transcription/PCR methods, the Micro-Fluidic Card requires less sample material (2 $\mathrm{ng}$ as opposed to $50 \mathrm{ng}$ of total RNA equivalents per reaction), only one-tenth the volume of TaqMan Universal Master Mix, and far less hands-on time. In fact, a full analysis, from total RNA extraction to obtaining the results, requires only 2 days.

We specifically designed this platform to simultaneously detect del/dup mutations affecting the exon composition/sequence (small mutations, any types) as well as expression of the DMD gene isoforms. Not only was this performed satisfactorily, but the FluiDMD was also found to be able to detect the decay of the DMD gene transcript induced by frame-shifting and/or stop mutations; RNA decay is a well-known phenomenon but has barely been studied in the dystrophin gene in terms of mechanism(s) or detection [Hamed and Hoffman, 2006; Tennyson et al., 1996]. It is, however, relevant to be detected, not only to improve understanding about functional consequences of dystrophin mutations in terms of transcript abundance, but also to consent monitoring of transcript stability which is an expected favorable event which may occur during the course of treatments aimed at reframing dystrophin, such as AONs (Antisense OligoNucleotides) or Ataluren.

As previously mentioned, FluiDMD was able to accurately identify already known and unknown deletions/duplications in the gene, and to directly explicate the pathogenic effect of mutations affecting splicing. This suggests that the array could be useful as a very accurate technique for large-scale molecular diagnosis of DMD.

For these reasons, we propose that FluiDMD could be used as a first-step screening method in the diagnostic procedure for DMD, if RNA is available, to be followed by MLPA and sequencing.

As expected, the FluiDMD was not able to directly identify stopcodon mutations and single-exon duplications, but it was, however, capable of detecting the resulting decay occurring along the DMD transcript, an indirect indicator of the presence of such mutations, which induce decay via frame alterations.

Moreover, FluiDMD was able to identify the out-of-frame duplication of exon 2, the most common duplication in the gene.

The exon 2 duplication in Patient 4 was indicated by FluiDMD as a delay in the CT value of exon junctions $1-2$ and 2-3, due to the competition of the two transcript species generated by the annealing of the forward and reverse primers to the duplicated regions. This is possible due to both the small size ( $62 \mathrm{bp}$ ) of exon 2 and the ability of the universal master mix to amplify fragments to the 150-bp limit (http://www3.appliedbiosystems.com/cms/groups/mcb_support/ ocuments/generaldocuments/cms_042996.pdf). As a result of the size of the DMD exons, the detection of single-exon duplications could be achieved in 12 of 79 exons $(2,4,19,61-64,66,71-73$, and 78 ) then allowing the direct detection of $53 \%$ of single-exon duplications as reported in the UMD-DMD database [Tuffery-Giraud et al., 2009].

As compared with other diagnostic tools such as MLPA and $\mathrm{CGH}$, the FluiDMD card has the advantage of identifying both the mutation and/or its pathogenic effect, thereby missing very few single-exon duplications. In addition, direct quantification of the junctions in $\mathrm{CNV}$ mutations confers greater sensitivity regarding reading frame with respect to both CGH and MLPA. In fact in the case of inversion of one or more exons, even within larger duplications, the FluiDMD would identify the resulting behavior of the transcript whereas CGH might or not identify CNV flanking the inversion site.

The failure to amplify the exon 48-49 junction, although being of no concern since the presence of exon 48 is granted by the amplification of junction 47-48, does raise the following issue: all mutations that fall in the sequences of the junction systems could impair or alter the efficiency of amplification of the targeted system and would therefore consent identification of the variation. This implies that even some point mutations could be directly identified by the FluiDMD card as occurred for the junction 19/20 in the DMD skin; validation will merely require the specific amplification and sequencing of the affected region.

The apparent ability of this method to fully characterize the DMD gene and directly record the behavior of almost all known gene mutations is significant for many reasons. The first is that spectrum of mutations accurately identified by this system is the broadest and most complete among the single tools available for diagnostic purposes to date, as it directly detects deletions, duplications (all multi exon and some single exon) and point splicing affecting mutations. The ability to detect the decay in the DMD transcript, a unique characteristic of our assay, also consents the indirect detection of all stop and out-of-frame mutations.

Moreover, if compared to the present RNA-based diagnostic procedures, which require large amounts of RNA and considerable hands-on time, this system promises to be the most comprehensive, quick, and easily performed. In fact it could be run in any laboratory with an Applied Biosystems 7900HT Real-Time machine, requiring a mere 2 days from biopsy to the results.

Interestingly, similar fluidic cards may represent a tool for monitoring both the maintenance of correct exon composition and the absence of off-target effects in exon-skipping therapies [Goemans et al., 2011; Kinali et al., 2009], and the amount of RNA decay, as a quantitative rapid alternative to the usual RT-PCR technique.

Furthermore, the FluiDMD is also able to detect the presence of all dystrophin isoforms in a very short time, a capacity that will certainly be of interest to researchers aiming to evaluate expression of its isoforms in various tissues/cells. Indeed, the significance of isoform profiling, since their retained function, may have a role in conferring functionality to the dystrophin network, has been reported by several authors [Fletcher et al., 2010].

Last, but by no means least, we demonstrated the validity of FluiDMD in detecting the dystrophin RNA profile in readily available tissues such as skin, which was successful not only in terms of painstakingly cultured and Myo-D-transformed cells, but also in whole tissue. This paves the way to the use of a low-invasive skin biopsy in dystrophin messenger profiling, a boon in both diagnostic circles, especially in circumstances where muscle tissue is unavailable or inadequate (quality and quantity), and in periodic monitoring of the dystrophin messenger during treatments.

In conclusion, although in this case the TLDA was successfully applied to the dystrophin gene, there appears to be no impediment 
to its purpose design for a variety of human and non-human genes (for example adapted to suitable animal models), and consequently it potentially harbors a vast range of applications in both diagnostics and research into other genetic diseases.

\section{Disclosure/Conflict of Interest}

All the authors declare no conflict of interest.

\section{References}

Abbs S, Tuffery-Giraud S, Bakker E, Ferlini A, Sejersen T, Mueller CR. 2010 Best practice guidelines on molecular diagnostics in Duchenne/Becker muscular dystrophies. Neuromuscul Disord 20:422-427.

Bennett RR, den Dunnen J, O'Brien KF, Darras BT, Kunkel LM. 2001. Identification of mutations in the dystrophin gene via automated DHPLC screening and direct sequencing. BMC Genet 2:17.

Bonnal RJ, Severgnini M, Castaldi A, Bordoni R, Iacono M, Trimarco A, Torella A, Piluso G, Aurino S, Condorelli G, De Bellis G, Nigro V. 2010. Reliable resequencing of the human dystrophin locus by universal long polymerase chain reaction and massive pyrosequencing. Anal Biochem 406:176-184.

Bovolenta M, Neri M, Fini S, Fabris M, Trabanelli C, Venturoli A, Martoni E, Bassi E, Spitali P, Brioschi S, Falzarano MS, Rimessi P, Ciccone R, Ashton E, McCauley J, Yau S, Abbs S, Muntoni F, Merlini L, Gualandi F, Ferlini A. 2008. A novel custom high density-comparative genomic hybridization array detects common rearrangements as well as deep intronic mutations in dystrophinopathies. BMC Genomics 9:572.

Bovolenta M, Rimessi P, Dolcini B, Ravani A, Ferlini A, Gualandi F. 2010. Prenatal diagnosis of Duchenne muscular dystrophy by comparative genomic hybridization. Clin Genet 77:503-506.

Chaouch S, Mouly V, Goyenvalle A, Vulin A, Mamchaoui K, Negroni E, Di Santo J, Butler-Browne G, Torrente Y, Garcia L, Furling D. 2009. Immortalized skin fibroblasts expressing conditional MyoD as a renewable and reliable source of converted human muscle cells to assess therapeutic strategies for muscular dystrophies: validation of an exon-skipping approach to restore dystrophin in Duchenne muscular dystrophy cells. Hum Gene Ther 20:784-790.

Deburgrave N, Daoud F, Llense S, Barbot JC, Récan D, Peccate C, Burghes AH, Béroud C, Garcia L, Kaplan JC, Chelly J, Leturcq F. 2007. Protein and mRNAbased phenotype-genotype correlations in $\mathrm{DMD} / \mathrm{BMD}$ with point mutations and molecular basis for BMD with nonsense and frameshift mutations in the DMD gene. Hum Mutat 28:183-195.

del Gaudio D, Yang Y, Boggs BA, Schmitt ES, Lee JA, Sahoo T, Pham HT, Wiszniewska J, Chinault AC, Beaudet AL, Eng CM. 2008. Molecular diagnosis of Duchenne/Becker muscular dystrophy: enhanced detection of dystrophin gene rearrangements by oligonucleotide array-comparative genomic hybridization. Hum Mutat 29:11001107.

Ferlini A, Sabatelli P, Fabris M, Bassi E, Falzarano S, Vattemi G, Perrone D, Gualandi F, Maraldi NM, Merlini L, Sparnacci K, Laus M, Caputo A, Bonaldo P, Braghetta P, Rimessi P. 2010. Dystrophin restoration in skeletal, heart and skin arrector pili smooth muscle of mdx mice by ZM2 NP-AON complexes. Gene Ther 17:432-438. Epub 2009 Nov 12.

Flanigan KM, Dunn DM, von Niederhausern A, Soltanzadeh P, Gappmaier E, Howard MT, Sampson JB, Mendell JR, Wall C, King WM, Pestronk A, Florence JM, Connolly AM, Mathews KD, Stephan CM, Laubenthal KS, Wong BL, Morehart PJ, Meyer A, Finkel RS, Bonnemann CG, Medne L, Day JW, Dalton JC, Margolis MK, Hinton VJ; United Dystrophinopathy Project Consortium, Weiss RB. 2009. Mutational spectrum of DMD mutations in dystrophinopathy patients: application of modern diagnostic techniques to a large cohort. Hum Mutat 30:1657-1666.

Flanigan KM, Dunn DM, von Niederhausern A, Soltanzadeh P, Howard MT, Sampson JB, Swoboda KJ, Bromberg MB, Mendell JR, Taylor L, Anderson CB, Pestronk A, Florence J, Connolly AM, Mathews KD, Wong B, Finkel RS, Bonnemann CG, Day JW, McDonald C, Weiss RB; the United Dystrophinopathy Project Consortium. 2011. Nonsense mutation-associated Becker muscular dystrophy: interplay between exon definition and splicing regulatory elements within the DMD gene. Hum Mutat. 32(3):299-308. (Epub ahead of print).

Flanigan KM, von Niederhausern A, Dunn DM, Alder J, Mendell JR, Weiss RB. 2003. Rapid direct sequence analysis of the dystrophin gene. Am J Hum Genet 72:931939.

Fletcher S, Adams AM, Johnsen RD, Greer K, Moulton HM, Wilton SD. 2010. Dystrophin isoform induction in vivo by antisense-mediated alternative splicing. Mol Ther 18:1218-1223.

Goemans NM, Tulinius M, van den Akker JT, Burm BE, Ekhart PF, Heuvelmans N, Holling T, Janson AA, Platenburg GJ, Sipkens JA, Sitsen JM, Aartsma-Rus A, van Ommen GJ, Buyse G, Darin N, Verschuuren JJ, Campion GV, de Kimpe SJ, van Deutekom JC. 2011. Systemic administration of PRO051 in Duchenne's muscular dystrophy. N Engl J Med 364:1513-1522.

Gualandi F, Neri M, Bovolenta M, Martoni E, Rimessi P, Fini S, Spitali P, Fabris M, Pane M, Angelini C, Mora M, Morandi L, Mongini T, Bertini E, Ricci E, Vattemi G, Mercuri E, Merlini L, Ferlini A. 2009. Transcriptional behaviour of DMD gene duplications in DMD/BMD males. Hum Mutat 30:E310-E319.

Hamed SA, Hoffman EP. 2006. Automated sequence screening of the entire dystrophin cDNA in Duchenne dystrophy: point mutation detection. Am J Med Genet B Neuropsychiatr Genet 141B:44-50.

Hegde MR, Chin EL, Mulle JG, Okou DT, Warren ST, Zwick ME. 2008. Microarraybased mutation detection in the dystrophin gene. Hum Mutat 29:10911099.

Janssen B, Hartmann C, Scholz V, Jauch A, Zschocke J. 2005. MLPA analysis for the detection of deletions, duplications and complex rearrangements in the dystrophin gene: potential and pitfalls. Neurogenetics 6:29-35.

Kesari A, Pirra LN, Bremadesam L, McIntyre O, Gordon E, Dubrovsky AL, Viswanathan V, Hoffman EP. 2008. Integrated DNA, cDNA, and protein studies in Becker muscular dystrophy show high exception to the reading frame rule. Hum Mutat 29:728-737.

Kinali M, Arechavala-Gomeza V, Feng L, Cirak S, Hunt D, Adkin C, Guglieri M, Ashton E, Abbs S, Nihoyannopoulos P, Garralda ME, Rutherford M, McCulley C, Popplewell L, Graham IR, Dickson G, Wood MJ, Wells DJ, Wilton SD, Kole R, Straub V, Bushby K, Sewry C, Morgan JE, Muntoni F. 2009. Local restoration of dystrophin expression with the morpholino oligomer AVI-4658 in Duchenne muscular dystrophy: a single-blind, placebo-controlled, dose-escalation, proof-ofconcept study. Lancet Neurol 8:918-928. Erratum in: Lancet Neurol. 2009 8:1083.

Lalic T, Vossen RH, Coffa J, Schouten JP, Guc-Scekic M, Radivojevic D, Djurisic M, Breuning MH, White SJ, den Dunnen JT. 2005 Nov; Deletion and duplication screening in the DMD gene using MLPA. Eur J Hum Genet 13:1231-1234.

Magri F, Del Bo R, D’Angelo MG, Govoni A, Ghezzi S, Gandossini S, Sciacco M, Ciscato P, Bordoni A, Tedeschi S, Fortunato F, Lucchini V, Cereda M, Corti S, Moggio M, Bresolin N, Comi GP. 2011. Clinical and molecular characterization of a cohort of patients with novel nucleotide alterations of the Dystrophin gene detected by direct sequencing. BMC Med Genet 11:12-37.

Monaco AP, Bertelson CJ, Liechti-Gallati S, Moser H, Kunkel LM. 1988. An explanation for the phenotypic differences between patients bearing partial deletions of the DMD locus. Genomics 2:90-95.

Muntoni F, Torelli S, Ferlini A. 2003. Dystrophin and mutations: one gene, several proteins, multiple phenotypes. Lancet Neurol 2:731-740.

Pichavant C, Aartsma-Rus A, Clemens PR, Davies KE, Dickson G, Takeda S, Wilton SD, WolffJA, Wooddell CI, Xiao X, Tremblay JP. 2011. Current status of pharmaceutical and genetic therapeutic approaches to treat DMD. Mol Ther 19:830-840.

Spitali P, Rimessi P, Fabris M, Perrone D, Falzarano S, Bovolenta M, Trabanelli C, Mari L, Bassi E, Tuffery S, Gualandi F, Maraldi NM, Sabatelli-Giraud P, Medici A, Merlini L, Ferlini A. 2009. Exon skipping-mediated dystrophin reading frame restoration for small mutations. Hum Mutat 30:1527-1534.

Tennyson CN, Shi Q, Worton RG. 1996. Stability of the human dystrophin transcript in muscle. Nucleic Acids Res 24:3059-3064.

Tuffery-Giraud S, Béroud C, Leturcq F, Yaou RB, Hamroun D, Michel-Calemard L, Moizard MP, Bernard R, Cossée M, Boisseau P, Blayau M, Creveaux I, GuiochonMantel A, de Martinville B, Philippe C, Monnier N, Bieth E, Khau Van Kien P, Desmet FO, Humbertclaude V, Kaplan JC, Chelly J, Claustres M. 2009. Genotypephenotype analysis in 2,405 patients with a dystrophinopathy using the UMDDMD database: a model of nationwide knowledgebase. Hum Mutat 30:934945. 